(1794), nom. superfl.; Valeriana ringens Salisb., Prodr. Stirp. Cahap. Allerton: 56 (1796), nom. superfl.; Mitrophora cornucopiae (L.) Raf., Chlor. Aetn.: 5 (1813); Fedia graciliflora sensu Lange in Willk. \& Lange, Prodr. Fl. Hispan. 2: 6 (1870), non Fisch. \& C.A. Mey. (1840); Fedia langei Pomel, Nouv. Mat. Fl. Atlant. 1: 73 (1874); Fedia scorpioides var. atropurpurea Maire in Bull. Soc. Hist. Nat. Afrique N. 20: 24 (1929)] reúne plantas en las que los frutos miden 2,6-5,4 x 4,4-5,2 $\mathrm{mm}$, son ovoideo-subesféricos y presentan escaso tejido esponjoso en sus cavidades estériles, careciendo además de eleosoma. De estas plantas, difieren las de la var. scorpioides (Dufresne) López \& Devesa, que presentan frutos 3-5,6 x 1,2-1,8 mm, oblongoideos 0 subcilíndricos, provistos de eleosoma y con abundante tejido esponjoso en sus cavidades estériles. Ambas coexisten en casi toda el área de distribución de la especie en el territorio peninsular ESP.: [(A) Ba, Ca, Co, Gr, H, J, Ma, Se. PORT.: AAl, Ag, (BAl), E, R].

Aceptado para su publicación en octubre de 2003

Dirección de los autores. Unidad de Botánica, Departamento de Biología y Producción Vegetal, Facultad de Ciencias, Universidad de Extremadura. Avda. de Elvas s/n. 06071 Badajoz.

\title{
130. NUEVAS COMBINACIONES EN EL GÉNERO GALIUM L.
}

\author{
Ana ORTEGA OLIVENCIA y Juan Antonio DEVESA ALCARAZ
}

New combinations in Galium.

Palabras clave. Galium, Península Ibérica, Rubiaceae, taxonomía.

Key words. Galium, Iberian Peninsula, Rubiaceae, taxonomy.

Galium saxatile var. vivianum (Kliphuis) Ortega-Olivencia \& Devesa, comb. \& stat. novo

$\equiv$ Galium hercynicum subsp. vivianum Kliphuis in Bot. Not. 125: 491 (1972) [basión.; Typus: M. BELIZ, GONCALVES \& RUIVO nr. 1263, 17.7.1953; Beira-Alta, Serra da Estrela, Portugal (LISU)].

$\equiv$ Galium vivianum (Kliphuis) Holub in Folia Geobot. Phytotax. 9: 273 (1974).

$\equiv$ Galium saxatile subsp. vivianum
(Kliphuis) Ehrend. in Pl. Syst. Evol. 124: 176 (1975).

Galium saxatile L., Sp. Pl.: 106 (1753) es una especie cuya área de distribución se extiende por Escandinavia y Europa central y atlántica (incluidas las Islas Azores), que se ha introducido también en el $\mathrm{N}$ de América y Argentina. En la Penínula Ibérica habita en cervunales, praderas de lugares húmedos y enclaves sombríos de bosques y matorrales, 
fundamentalmente en sustrato granítico, pizarroso o cuarcítico, muy rara vez en calizo, localizándose en las montañas del N, NW y C [(And.) Esp.: Av Bu C Cc Ge? (Gu) (Hu) Le (Lo) Lu Na O Or (P) Po S (Sg) Sa SS So (Vi) Za. Port.: AAl BA BB BL Mi TM].

En su seno se reconocen dos pautas de variación basadas, fundamentalmente, en la longitud de los entrenudos medios y superiores de los tallos y en el tamaño de las hojas, si bien son muy frecuentes individuos que comparten caracteres de ambas. Por un lado, Galium saxatile var. saxatile, ampliamente distribuida por toda el área de distribución peninsular, reúne los individuos tetraploides $(2 n=44)$, por lo general laxamente cespitosos, con tallos 6,5-40 cm x 0,5-0,8(1) mm y entrenudos mayores (14)21-75 mm. Las hojas suelen ser desiguales, las de los tallos estériles $(1,5) 3,5$ $9,5 \times(0,8) 1-3,3 \mathrm{~mm}$, aparecen dispuestas en verticilos de 4-6/nudo y en general son obovadas, obtusas y cortamente aristuladas o bien \pm oblanceoladas y agudas; las basales de los tallos fértiles son semejantes, aunque a menudo están secas durante la floración; las medias y superiores $(3,5) 6-12 \times(0,9) 1,3-3(3,4)$ mm, en verticilos de 5-7(8), estrecha o anchamente oblanceoladas y agudas, menos frecuentemente obovadas $\mathrm{y} \pm$ obtusas.

Por otro lado, las plantas diploides $(2 \mathrm{n}=$ 22), se asimilan a Galium saxatile var. vivianum (Kliphuis) Ortega-Olivencia \& Devesa, con distribución peninsular algo más restringida [Esp.: Av C (Cc) Le Na O Or Sa SS Za. Port.: BA Mi TM]. Esta variedad reúne plantas densamente cespitosas, con tallos 2,5-15(20) $\mathrm{cm} \times$ 0,2-0,4(0,55) mm y entrenudos 3-15(40) $\mathrm{mm}$; las hojas son muy pequeñas, las de los tallos estériles 1,5-5(6) x 0,6-2,1 mm, en verticilos de 4-6/nudo, obovadas, anchamente elípticas o subrómbicas, las basales de los tallos fértiles semejantes, en general secas durante la floración, y las medias y superiores 2-6(6,8) x 0,6-1,7 mm, en verticilos de (4)5-7, estrecha o anchamente oblanceoladas o linearelípticas, muy rara vez obovadas.
Galium papillosum subsp. helodes (Hoffmanns. \& Link) Malag. ex OrtegaOlivencia \& Devesa, comb. nova.

$\equiv$ Galium helodes Hoffmanns. \& Link, $F l$. Portug. 2: 47-48 (1820-1824) [basión.; Ind. loc.: "Dans les lieux marécageux aux environs de Torres-vedras"].

$\equiv$ Galium pumilum var. helodes (Hoffmanns. \& Link) P. Monts. in Collect. Bot. (Barcelona) 5: 646 (1958), comb. inval.

$\equiv$ Galium uliginosum [B] "elodes" (Hoffmanns. \& Link) Lange in Willk. \& Lange, Prodr. Fl. Hispan. 2: 321 (1868).

$\equiv$ Galium papillosum subsp. helodes (Hoffmanns. \& Link) Malag., Sin. Fl. Iber. 67: 1065 (1977), comb. inval.

$=\therefore$ Galium uliginosum subsp. [b] langei Cout., Notas Fl. Port. 7: 7 (1930) [Ind. loc.: “ Trás-os-Montes (Bragança) e Minho (Melgaço)"]. = Galium langei (Cout.) Pinto da Silva, Agron. Lusit. 34(3): 194 (1972, publ. 1973), non Galium langei Sennen (1929).

= Galium uliginosum sensu auct. hisp. \& lusit., non L. (1753).

= Galium "elodes" auct.

Galium papillosum Lapeyr., Hist. Pl. Pyrénées: 66 (1813) se distribuye por la Península Ibérica -Portugal, Cordillera Cantábrica, Pirineos, Sistema Ibérico, Sistema Central y Sierra de Segura-Cazorla- y SE de Francia, habitando en el territorio peninsular en herbazales de bosques perennifolios y caducifolios, riberas y taludes, gleras y roquedos, sobre sustratos ácidos o básicos [And. Esp.: Ab Av B Bi Bu C Cc Cs Cu Ge Gr Gu Hu J L Le Lo Lu M Na O Or P Po (S) Sa Sg So T Te Va Z Za. Port.: Ag BA BB BL DL E Mi R TM]. Se trata de una especie extremadamente polimorfa para la que se han descrito numerosos taxones, en su mayoría meros ecótipos que se localizan en los distintos sistemas montañosos del territorio, en el que se reconocen dos subespecies.

Galium papillosum Lapeyr. subsp. papillosum está distribuida por el $\mathrm{N}$ de Portugal, 
Cordillera Cantábrica, Pirineos, Sistema Ibérico, Sistema Central y Complejo SeguraCazorla [And. Esp.: Ab Av B Bu Cc Cs Cu Ge Gr Gu Hu J L Le Lo Lu M Na O Or P S Sa Sg So $\mathrm{T}$ Te Va Z Za. Port.: BA Mi TM]. Se caracteriza porque sus individuos poseen tallos por lo general más o menos fuertes, aculeados, papiliformes o hirsutos; hojas casi siempre subcoriáceas, en verticilos de (5)6-9(11); inflorescencia con ramas delgadas, rara vez capilares y pedicelos florales $0,1-0,15(0,2) \mathrm{mm}$ de grosor, generalmente menores que el diámetro de la corola. Por el contrario, Galium papillosum subsp. helodes (Hoffmanns. \& Link) Malag. ex Ortega-Olivencia \& Devesa se restringe al $\mathrm{W}$ de la Península Ibérica (Portugal y Galicia) [Esp.: C Lu Or Po. Port.: Ag BA BB BL DL E Mi R TM], y sus plantas presentan tallos delicados, aculeados pero nunca hirsutos; hojas débiles y delicadas, en verticilos de 4-6(7); inflorescencia con ramas capilares y pedicelos florales $0,05-0,1 \mathrm{~mm}$ de grosor, generalmente mayores que el diámetro de la corola. En algunas poblaciones de ambas subespecies es frecuente que sus tallos posean los entrenudos lisos o poco aculeados.

Galium estebani var. leioclados (Pau) OrtegaOlivencia \& Devesa, comb. \& stat. novo

$\equiv$ Galium silvestre var. curtifolium $\mathrm{f}$. leioclados Pau in Bol. Soc. Aragonesa Ci. Nat. 9: 59 (1910) [basión.; Ind. loc.: "Pozondón, Orihuela y Sierra alta (Bronchales)". Lectotypus: "Caroli Paui herbarium hispanicum (impr.) 117684 (impr.)/ Galium silvestre Poll. (manuscr.) / var. ) leioclados Pau (manuscr.) / Inter Pozondon et Orihuela del Tremedal. (manuscr.) / Legi (impr.) 19.VI.1907 (manuscr.)" (seleccionado aquí!)].

= Galium pinetorum Ehrend. in Sitzungsber. Kaiserl. Akad. Wiss., Math.Naturwiss. Cl., Abt. 1, 169: 410-411 (1960) [Ind. loc.: "Hispania, prov. Teruel: Sierra d'Albarracin (Sierra del Pinar), bois de pins, sur le calcaire, 1500 m; E. REVERCHON, 6.
1895 et 1896, no. 664 (.G. valentinum”. Holotypus: WU; Isotypus: W, GZU, G, P, LY, M, PR, GR, BP, FI, MA, E].

= Galium pumilum Murray subsp. pinetorum (Ehrend.) Vigo, Veg. Massís Penyagolosa, Inst. Est. Catalans, Secc. Ciènc. 37: 91 (1968).

$\equiv$ Galium silvestre var. leioclados $\mathrm{Pau}$, nom. in sched. (MA 117684 y 117685).

= Galium silvestre f. mayor Zubia, nom in sched. (MA 117651).

= Galium pinetorum subsp. cantabricum Ehrendf., nom. in sched.

= Galium chlorophyllum sensu Sennen, non Baillet \& Timb.-Lagr. (1862).

= Galium valentinum Lange sensu auct. hisp. p.p.

= Galium silvestre sensu auct. hisp., non Pollich (1776-1777).

= Galium pumilum subsp. vulgatum auct.

cat., non Schinz \& R. Keller (1914).

= Galium pumilum auct. hisp. \& lusit.

= Galium idubedae sensu auct. hisp., non (Pau ex Debeaux) Pau ex Ehrend. (1960).

Galium estebani Sennen, Diagn. Nouv.: 289 (1936) [= Galium silvestre var. curtifolium Pau in Bol. Soc. Aragonesa Ci. Nat. 9: 59 (1910)] habita en bosques perennifolios y caducifolios, matorrales y prados, grietas de rocas y herbazales de bordes de camino, preferentemente en sustratos básicos (calizas, dolomías, yesos o margas yesíferas), de la mitad $N$ y $S E$ de España y $S$ de Francia (Corbières) [Esp.: Al B Bu Cs Cu Ge Gr Gu Hu J L Le Lo Lu Na O Or P S Sa Sg So Te Va Vi]. Presenta en el territorio de Flora Iberica dos variedades, que conviven en muchas situaciones.

Galium estebani Sennen var. estebani reúne plantas hirsutas o incluso tomentosas [Esp.: B Bu Cu Ge Gr Gu Hu L Le Lo Lu Or P So Te Va], con pelos 0,1-0,4 $\mathrm{mm}$ en las hojas -sobre todo las del tercio basal- y al menos también en las zonas basales de los tallos, 
disminuyendo progresivamente hacia la zona florífera. Por el contrario, Galium estebani var. leioclados Ortega-Olivencia \& Devesa incluye plantas con entrenudos glabros y lisos, y hojas con haz antrorso-escabriúscula o glabrescente, envés glabro y márgenes variadamente papiliformes o escabriúsculos. Esta variedad se encuentra también ampliamente distribuida [Esp.: Al B Bu Cs Cu $\mathrm{Ge} \mathrm{Gr} \mathrm{Gu} \mathrm{Hu} \mathrm{J} \mathrm{L} \mathrm{Le} \mathrm{Lo} \mathrm{Lu} \mathrm{Na} \mathrm{O} \mathrm{Or} \mathrm{P} \mathrm{S} \mathrm{Sa} \mathrm{Sg}$ So Te Va Vi], y en algunas zonas (provincias de Cuenca, Logroño y Zaragoza) sus individuos presentan características transicionales hacia Galium idubedae (Pau ex Debeaux) Pau ex Ehrend., pudiendo tratarse tal vez de plantas de origen híbrido.

\section{Galium aparine subsp. spurium var. aparinella}

(Lange) Ortega-Olivencia \& Devesa, comb. \& stat. novo

$\equiv$ Galium aparinella Lange, Index Sem. Hort. Haun.: 24 (1855) [basión.; Ind. loc.: "Inter suffrutices (Frankenia thymifolia, Centaurea hypssopifolia cet.) solo gypsaceo ad Aranjuez lect. 21 Mai 1852". Isotypus: MA 118166, BC 97299 (seleccionados aquí!).

E Galium spurium subsp. aparinella (Lange) Rivas Mart. \& Castroviejo in Rivas Mart., Anales Inst. Bot. Cavanilles 34: 555 (1978).

E Galium aparine subsp. aparinella (Lange) Jauzein, Fl. Champs Cult.: 863 (1995).

= Galium parisiense var. vestitum sensu auct. hisp., non Gren. \& Godr. (1850).

Galium aparine subsp. spurium (L.) Hartman, Svensk och Norsk Exc.-Fl.: 23 (1846) es un taxon ruderal y arvense e indiferente al sustrato, que se distribuye por Eurasia (posiblemente introducida en Islas Británicas), Región Mediterránea, Macaronesia (Canarias) y $\mathrm{N}$ de América. Está representado en gran parte de la Península Ibérica e Islas Baleares (Mallorca) [Esp.: A Ab Al Av B Ba Bu Cc Co CR Cs Cu Ge Gr Gu H Hu J L Le Lu M Ma O
Or PM [Mll] Sa Se Sg So T Te To V Va ViZZa. Port.: AAl BA BB BL DL Mi TM], en donde se reconocen tres variedades.

Galium aparine subsp. spurium (L.) Hartman var. spurium (L.) Wimm. \& Grab., Fl. Siles. 1: 120 (1827), con distribución muy restringida [Esp.: J L], incluye las plantas con frutos glabros. Galium aparine subsp. spurium var. infestum (Waldst. \& Kit.) Schübler \& Martens, Fl. Würtemb.: 103 (1834), ampliamente distribuida [Esp.: A Ab Al Av B Ba Bu Cc Co CR Cs Cu Ge Gr Gu (H) Hu J L Le Lu M Ma O Or Se Sg So T Te To V Va Vi Z Za. Port.: AAl BA BB BL DL Mi TM], incluye plantas robustas, con tallos $0,9-1,7$ $\mathrm{mm}$ de grosor, hojas y brácteas (5)17-38(48) x $(0,5) 1-3(3,8) \mathrm{mm}$, por lo general planas, inflorescencias con cimas multifloras y mericarpos setosos de hasta $2,1(2,5) \mathrm{mm}$. También con mericarpos setosos es Galium aparine subsp. spurium var. aparinella (Lange) Ortega-Olivencia \& Devesa, mucho menos extendida [Esp.: (A) Al Cs CR Gr Hu J L M Ma PM[MIl] Sa Se? To? V Va Z], que comprende los individuos más gráciles, con tallos 0,4-1,1 $\mathrm{mm}$ de grosor, hojas y brácteas 3-10(20) x 0,4$1,4(1,7) \mathrm{mm}$, lineares o muy estrechamente oblanceoladas, planas o más generalmente revolutas, con nervio y margenes normalmente engrosados, inflorescencias por lo general paucifloras (1-4 flores) y mericarpos 0,75 $1,5(1,7) \mathrm{mm}$. Entre la var. infestum y la var. aparinella existen muchas formas de transición.

AGRADECIMIENTOS. Nuestro sincero agradecimiento a los conservadores de los herbarios BC, COI, GDA-GDAC, JAEN, MA, MAF, MGC, SEV y UNEX. Trabajo financiado por el Ministerio de Ciencia y Tecnología a través de los proyectos Flora Iberica V y VI (PB96-0447 y REN2002-04634C05-04, respectivamente).

Dirección de los autores. Unidad de Botánica, Departamento de Biología y Producción Vegetal, Facultad de Ciencias, Universidad de Extremadura. Avda. de Elvas s/n. 06071 Badajoz. 